\title{
Predicting Symptom Relief After Reoperation for Suspected Internal Herniation After Laparoscopic Roux-en-Y Gastric Bypass
}

\author{
Leontine H. Wijngaarden ${ }^{1}$ (ID - Sophie L. van Veldhuisen ${ }^{2} \cdot$ René A. Klaassen $^{1}$ • Erwin van der Harst ${ }^{1}$. \\ Charles C. van Rossem ${ }^{1}$ • Ahmet Demirkiran ${ }^{2}$. Steve M. M. de Castro ${ }^{3}$ - Frederik H. W. Jonker ${ }^{1}$
}

Published online: 18 July 2018

(C) The Author(s) 2018

\begin{abstract}
Background Internal herniation $(\mathrm{IH})$ is one of the most common long-term complications after laparoscopic Roux-en-Y gastric bypass (LRYGB). Diagnosis of IH may be difficult, and not all patients with suspected IH will have full relief of symptoms after closure of both mesenteric defects.

Objectives To investigate possible predictive factors for relief of symptoms in patients with suspected IH.

Methods All patients that underwent reoperation for (suspected) IH after LRYGB from June 2009 to December 2016 were retrospectively evaluated in this multicentre cohort study. Logistic regression analysis was used to identify predictive factors for pain relief after closure of the mesenteric defects.

Results A total of 193 patients underwent laparoscopy for (suspected) IH during the study period. The median interval between LRYGB and reoperation was $18.3 \pm 19.0$ months. In $40.2 \%$ of cases, IH was identified on computed tomography (CT), and IH was objectified during surgery in $61.1 \%$. Postoperative symptom relief was observed in 146 patients $(77.2 \%)$. For patients in which $\mathrm{IH}$ was present during surgery, $82.8 \%$ had relief of pain postoperatively, as compared to $68.5 \%$ for those procedures in which no IH was found. The only significant predictor for postoperative pain relief was a swirl sign on CT (OR 4.24, 95\%CI 1.63-11.05).

Conclusions Pain relief after closure of the mesenteric defects for IH remains unpredictable. A positive CT for IH was a predictive factor for symptom relief after reoperation for (suspected) IH after LRYGB. However, many patients benefit from closure of the mesenteric defects, irrespective of perioperative presence of $\mathrm{IH}$.
\end{abstract}

Keywords Internal herniation $\cdot$ Laparoscopic Roux-en-Y gastric bypass $\cdot$ Mesenteric defect closure

\section{Introduction}

The laparoscopic Roux-en-Y gastric bypass (LRYGB) has become a common bariatric procedure leading to satisfying long-term results in both weight reduction and reduction or even remission of comorbidities of morbid obesity [1-4]. However, due to altered bowel anatomy after LRYGB, internal herniation $(\mathrm{IH})$ can occur through either the mesenteric
Leontine H. Wijngaarden

k.wijngaarden@hotmail.com

Sophie L. van Veldhuisen

s.l.vanveldhuisen@gmail.com

René A. Klaassen

klaassenr@maasstadziekenhuis.nl

Erwin van der Harst

harste@maasstadziekenhuis.nl

Charles C. van Rossem

rossemc@maasstadziekenhuis.nl
Ahmet Demirkiran

ademirkiran@ rkz.nl

Steve M. M. de Castro

s.m.m.decastro@olvg.nl

Frederik H. W. Jonker

jonkerfrederik@hotmail.com

1 Department of Surgery, Maasstad Hospital, Maasstadweg 21, 3079

DZ Rotterdam, The Netherlands

2 Department of Surgery, Rode Kruis Hospital, Beverwijk, The Netherlands

3 Department of Surgery, OLVG, Amsterdam, The Netherlands 
defect of Petersen's space or the mesenteric defect of the jejunojejunostomy (JJ-stomy) during follow-up [5]. The reported incidence of internal herniation varies widely between 1.6 and $9.3 \%$ [5-9]. The typical presentation of patients with an internal herniation is intermittent, postprandial, upper abdominal pain, sometimes accompanied by nausea and vomiting $[10,11]$. Less frequently acute intestinal obstruction with or without bowel strangulation may occur, in which case emergency surgery is indicated. The mean interval between LRYGB and presentation of IH varies between 15 and 26 months in larger series [12, 13].

The presence of a 'swirl sign', caused by rotation of the mesenteric vessels on computed tomography $(\mathrm{CT})$, is the golden standard to diagnose an $\mathrm{IH}$, albeit varying sensitivity outcomes of CT have been reported [14-16]. Typically, management of IH consists of a reoperation with repositioning of the herniating bowel and closure of both mesenteric defects $[17,18]$.

Since IH may present with non-specific symptoms, preoperative diagnosis may be difficult and negative explorations have been described [8]. In some patients with typical intermittent pain symptoms and a clear 'swirl sign' on CT scan, actual visible IH may be absent during surgery. In case of open mesenteric defects without objectified $\mathrm{IH}$, patients may still benefit from closure of the mesenteric defects. Even more strikingly, some asymptomatic patients may have IH clearly visible on abdominal $\mathrm{CT}$ or during reoperation but do not benefit from closure. Therefore, outcome of pain and symptom relief after mesenteric defect closure seems to be highly unpredictable in literature.

The aim of this study is to investigate patient-related factors and intraoperative findings in patients with delayed closure of mesenteric defects, in order to predict postoperative symptom relief after reoperation in patients with suspected $\mathrm{IH}$ after LRYGB.

\section{Methods}

\section{Patient Selection}

Mesenteric defects were not routinely closed during LRYGB at our institutions until January 2017. Generally, we differentiate between patients readmitted with acute symptoms of $\mathrm{IH}$, possibly with abdominal tenderness and hemodynamic instability, and patients with a more chronic or intermittent presentation. In case of acute symptoms, urgent abdominal CT is typically performed, with subsequent laparoscopy if $\mathrm{IH}$ is suspected or if serious abdominal symptoms persist without any diagnosis. Patients with a more chronic or intermittent presentation first undergo treatment with increased dosage of proton-pump inhibitors (PPI) and mucosal protective drugs (MPD). If complaints are persistent, an

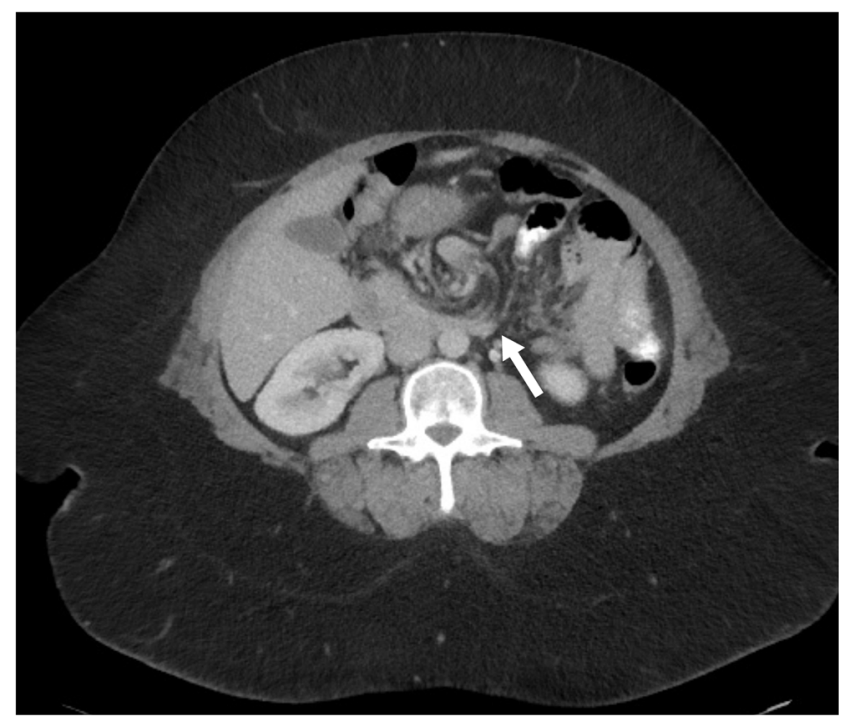

Fig. 1 Transverse CT scan through mesentery shows a subtle swirl sign of the mesenteric vessels of a 45-year-old woman with internal herniation

abdominal CT, gastroscopy (EGD), and/or reoperation are performed for suspected IH. For this analysis, all patients who underwent reoperation after LRYGB for suspected acute or chronic IH from June 2009 until December 2016 at the three bariatric institutions were

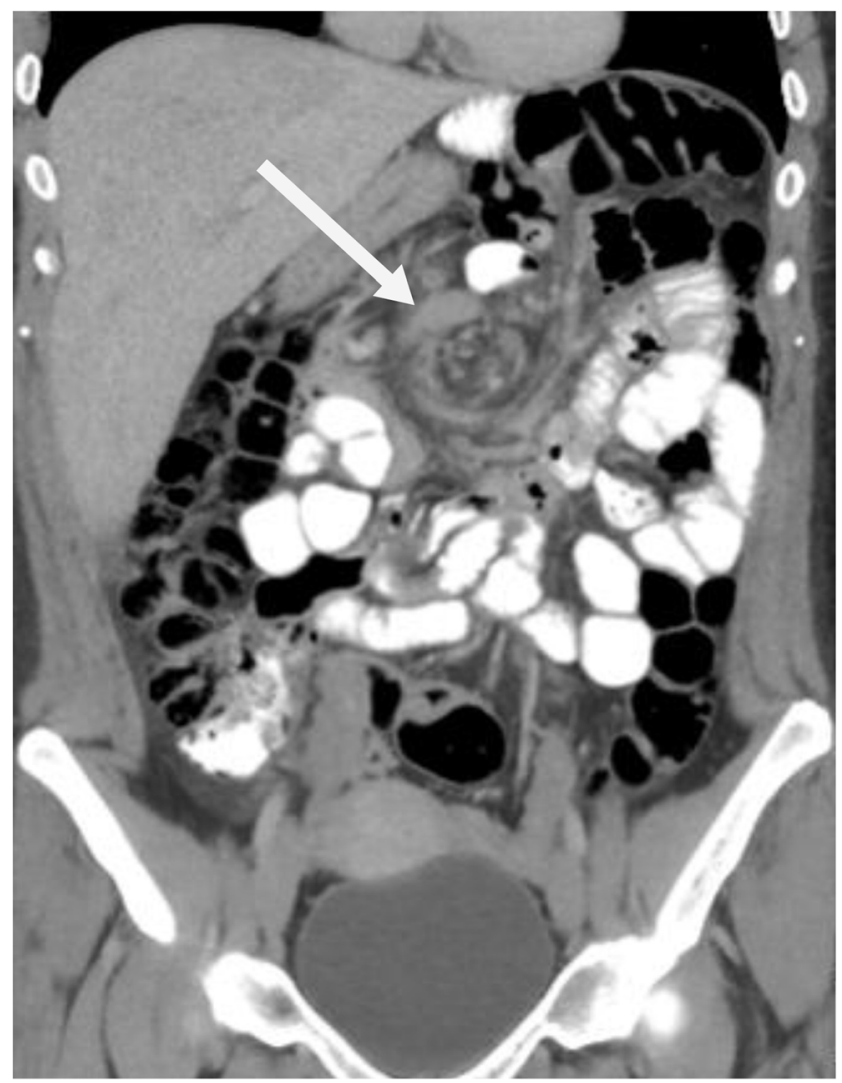

Fig. 2 Sagittal CT scan of a $360^{\circ}$ swirl sign in the mesenteric vessels of a 34-year-old woman with internal herniation 
Table 1 Baseline characteristics

\begin{tabular}{|c|c|c|}
\hline Variable & Number/mean & Range/percentage \\
\hline \multicolumn{3}{|l|}{ Demographics } \\
\hline Age at reoperation (years) & $41.5 \pm 9.6$ & $21-61$ \\
\hline Female gender & 171 & $88.6 \%$ \\
\hline BMI before gastric bypass ${ }^{1}$ & $42.4 \pm 5.4$ & $26-63$ \\
\hline BMI at reoperation ${ }^{1}$ & $28.9 \pm 5.7$ & $19-56$ \\
\hline BMI loss between LRYGB and reoperation ${ }^{1}$ & $13.6 \pm 5.3$ & $0-27$ \\
\hline$\% \mathrm{TWL}$ & $31.4 \pm 11.4$ & $-9.9-54.4$ \\
\hline Interval LRYGB and reoperation (months), median & $18.3 \pm 19.0$ & $0-99$ \\
\hline \multicolumn{3}{|l|}{ Presentation and imaging } \\
\hline Acute presentation & 72 & $37.3 \%$ \\
\hline CT abdomen performed & 145 & $74.6 \%$ \\
\hline CT normal & 87 & $60.4 \%$ \\
\hline Internal herniation on $\mathrm{CT}$ & 58 & $40.2 \%$ \\
\hline Days between CT and reoperation, median & $9.5 \pm 97.1$ & $0-535$ \\
\hline Days between IH on CT and reoperation, median & $1.0 \pm 21.4$ & $0-135$ \\
\hline \multicolumn{3}{|l|}{ Medical history } \\
\hline PPI use & 124 & $64.2 \%$ \\
\hline Smoking & 52 & $26.9 \%$ \\
\hline \multicolumn{3}{|l|}{ Previous cholecystectomy } \\
\hline No & 114 & $59.1 \%$ \\
\hline Before LRYGB & 21 & $10.9 \%$ \\
\hline After LRYGB but before reoperation & 31 & $16.1 \%$ \\
\hline During reoperation & 19 & $9.8 \%$ \\
\hline After reoperation & 8 & $4.1 \%$ \\
\hline \multicolumn{3}{|l|}{ Gastroscopy } \\
\hline No & 97 & $50.3 \%$ \\
\hline Before reoperation & 68 & $35.2 \%$ \\
\hline After reoperation & 21 & $10.9 \%$ \\
\hline Before and after reoperation & 7 & $3.6 \%$ \\
\hline
\end{tabular}

$\% \mathrm{TWL}=$ percentage total weight loss

$B M I$, body mass index; $L R Y G B$, laparoscopic Roux-en-Y gastric bypass; $C T$, computed tomography; $I H$, internal herniation; $P P I$, proton-pump inhibitor

${ }^{1}$ In $\mathrm{kg} / \mathrm{m}^{2}$

retrospectively reviewed. Patients were excluded if other abnormalities were found during reoperation that could explain the complaints (e.g., obstructing adhesions) or in case mesenteric defects turned out to be closed during exploration (e.g., by adhesions).

All CT scans were interpreted both by a radiologist and an experienced bariatric surgeon. We defined a CT scan as positive if a swirl sign with an estimated amount of swirl of at least $180^{\circ}$ was seen. Two examples of a positive swirl sign on CT are shown in Figs. 1 and 2. Intraoperative findings were investigated and postoperative pain relief was assessed for all patients. Subsequently, predictors of internal herniation during reoperation and predictors of postoperative pain relief were investigated. Pain relief was scored positive if the patient did not have postprandial, upper abdominal pain 3 months after reoperation.

\section{Surgical Procedure}

In our institutions, LRYGB was performed without primary closure of the mesenteric defects. The LRYGB was performed with an antecolic approach. After division of the jejunum, the biliopancreatic limb was positioned to the left of the alimentary limb while rotating the alimentary limb to the right. The jejunojejunal limb and biliopancreatic limb were positioned to the left of the alimentary limb.

Reoperations were performed by an experienced bariatric surgeon using laparoscopy with inspection of the bowel anatomy of the alimentary limb, the biliopancreatic limb, and the common limb. If internal herniation in Petersen's space or through the defect of the jejunojejunostomy (JJ-stomy) was present, the bowel was repositioned and the mesenteric defects 
Table 2 Operative characteristics

\begin{tabular}{|c|c|c|}
\hline Variable & Number $(n=193)$ & $\%$ \\
\hline Conversion/laparotomy & 6 & 3.1 \\
\hline Internal herniation during surgery & 118 & 61.1 \\
\hline Petersen's space hernia & 54 & 45.8 \\
\hline JJ-stomy hernia & 55 & 46.6 \\
\hline Peterson's and JJ-stomy & 9 & 7.6 \\
\hline \multicolumn{3}{|l|}{ Closure technique $^{1}$} \\
\hline Non-absorbable suture & 164 & 85.0 \\
\hline Staples & 22 & 11.4 \\
\hline \multicolumn{3}{|l|}{ Postoperative symptoms } \\
\hline Relief of symptoms ${ }^{2}$ & 146 & 77.2 \\
\hline \multicolumn{3}{|l|}{ Additional reoperations } \\
\hline For suspected recurrence IH & 37 & 19.2 \\
\hline Proven recurrence $\mathrm{IH}$ & 19 & 9.8 \\
\hline
\end{tabular}

JJ-stomy, jejunojejunostomy; $I H$, internal herniation

${ }^{1}$ In seven patients, absorbable sutures were used

${ }^{2}$ Missing data of 4 patients, so total population to answer this question is 190 patients

were either closed with running, non-absorbable sutures or with non-absorbable staples (EndoHernia ${ }^{\mathrm{TM}}$, Medtronic, Minneapolis, MN), depending on the surgeon's preference.

\section{End Points and Statistical Analysis}

Statistical analysis was performed with IBM SPSS Statistics, version 23 (SPSS, Chicago, IL). Univariate logistic regression was used to investigate predictors of $\mathrm{IH}$ during reoperation and postoperative pain relief. Crosstabs were used to calculate sensitivity and specificity of the interpretation of the CT scan. We have used LOWESS local regression to determine the point on which \%TWL could be a predictor for pain relief. Multivariate logistic regression was performed to determine independent predictors for pain relief. A $p$ value $<0.05$ was considered statistically significant.

\section{Results}

A total of 193 reoperations were performed for (suspected) IH, with an estimated incidence of $2.8 \%$ (a total of 6896 LRYGB were performed in our institutions during the study period). Mean age of patients at reoperation was $41.5 \pm 9.6$ years and $171(88.6 \%)$ patients were female. The median interval between gastric bypass and reoperation was $18.3 \pm 19.0$ months. Laparoscopic cholecystectomy was performed between LRYGB and reoperation for suspected IH in $16.0 \%$ of the study cohort (Table 1). In $35.2 \%$ of the patients, gastroscopy was performed before reoperation for suspected IH. In 28 (14.5\%) patients, gastroscopy was performed after reoperation; in 4 cases, a marginal ulcer was found.

Of all procedures, $72(37.3 \%)$ were performed in an acute setting. Preoperative abdominal CT was performed in 144 patients, and in 56 patients (38.9\%), signs of internal herniation were found. The sensitivity of the swirl sign found on CT for suspected IH after interpretation of a radiologist and an experienced bariatric surgeon was $50.0 \%$, and the specificity was $83.0 \%$.

An IH was found intraoperatively in 118 (61.1\%) patients; there was no preference for the JJ-stomy or Peterson's space (Table 2). There were no cases of intestinal ischemia. In 75 (38.9\%) patients, no abnormalities were found intraoperatively. Nevertheless, mesenteric defects were closed in these patients in order to prevent future $\mathrm{IH}$ - absorbable sutures were used more frequently than staples to close the mesenteric defects (164 vs.
Table 3 Predictors of intraoperative presence of internal herniation

\begin{tabular}{lcccc}
\hline Variable & Internal herniation $(\%)$ & OR & $95 \% \mathrm{CI}$ & $p$ value \\
\hline Age $>45(n=75)$ & $46(61.3 \%)$ & 1.01 & $0.56-1.84$ & 0.965 \\
Female gender $(n=171)$ & $101(59.1 \%)$ & 0.42 & $0.15-1.20$ & 0.107 \\
Male gender $(n=22)$ & $17(77.3 \%)$ & 2.36 & $0.83-6.69$ & 0.107 \\
Reoperation after 2014 $(n=137)$ & $87(64.0 \%)$ & 1.49 & $0.80-2.79$ & 0.214 \\
BMI $\geq 30$ at reoperation $(n=73)$ & $47(65.3 \%)$ & 1.34 & $0.73-2.46$ & 0.340 \\
BMI loss $\geq 20(n=25)$ & $15(60.0 \%)$ & 1.04 & $0.45-2.43$ & 0.926 \\
$\%$ TWL $>40(n=44)$ & $30(68.2 \%)$ & 1.54 & $0.75-3.14$ & 0.753 \\
Acute surgery $(n=73)$ & $57(79.2 \%)$ & 3.74 & $1.91-7.31$ & $<0.001 *$ \\
Elective surgery $(n=121)$ & $61(50.4 \%)$ & 0.27 & $0.14-0.52$ & $<0.001^{*}$ \\
CT normal $(n=87)$ & $43(47.3 \%)$ & 0.20 & $0.09-0.45$ & $<0.001^{*}$ \\
Internal herniation on CT $(n=58)$ & $48(82.8 \%)$ & 4.91 & $2.11-10.93$ & $<0.001^{*}$ \\
\hline
\end{tabular}

$\% \mathrm{TWL}=$ percentage total weight loss

$B M I$, body mass index; $G B$, gastric bypass; $C T$, computed tomography

*Significant difference 
Table 4 Predictors of symptom relief after reoperation

\begin{tabular}{lcccc}
\hline Variable & Symptom relief $(\%)$ & OR & 95\%CI & $p$ value \\
\hline Age $>45(n=73)$ & $56(76.7 \%)$ & 0.95 & $0.47-1.91$ & 0.889 \\
Female gender $(n=167)$ & $127(76.0 \%)$ & 0.50 & $0.14-1.78$ & 0.286 \\
Male gender $(n=22)$ & $19(86.4 \%)$ & 2.00 & $0.56-7.09$ & 0.286 \\
\% TWL $>40(n=44)$ & $34(77.0 \%)$ & 1.02 & $0.46-2.28$ & 0.962 \\
Acute surgery $(n=70)$ & $57(81.4 \%)$ & 1.48 & $0.71-3.07$ & 0.295 \\
Elective surgery $(n=119)$ & $89(74.8 \%)$ & 0.68 & $0.33-1.41$ & 0.295 \\
Smoking $(n=50)$ & $41(82.0 \%)$ & 1.48 & $0.65-3.34$ & 0.352 \\
CT normal $(n=86)$ & $57(66.3 \%)$ & 0.24 & $0.09-0.61$ & $0.003 *$ \\
Internal herniation on CT $(n=56)$ & $50(89.3 \%)$ & 4.24 & $1.63-11.05$ & $0.003 *$ \\
Internal herniation during surgery $(n=116)$ & $96(82.8 \%)$ & 2.21 & $1.11-4.40$ & $0.024 *$ \\
Petersen's space $(n=53)$ & $42(79.2 \%)$ & 1.18 & $0.54-2.55$ & 0.683 \\
JJ-stomy $(n=54)$ & $45(83.3 \%)$ & 1.68 & $0.75-3.80$ & 0.210 \\
Petersen's space and JJ-stomy ${ }^{1}(n=9)$ & $9(100 \%)$ & & & 0.095 \\
Closing technique & & & & \\
Non-absorbable suture $(n=160)$ & $123(76.9 \%)$ & 0.78 & $0.28-2.22$ & 0.645 \\
Staples $(n=22)$ & $17(77.3 \%)$ & 1.00 & $0.35-2.89$ & 0.998 \\
\hline
\end{tabular}

$\% \mathrm{TWL}=$ percentage total weight loss

$B M I$, body mass index; $G B$, gastric bypass; $C T$, computed tomography; $J J$-stomy, jejunojejunostomy

* Significant difference

${ }^{1}$ As all patients with an internal herniation at both spaces had postoperative symptom relief, odds ratio could not be calculated and therefore Pearson's chi-square was used
22). In the remaining 7 patients, absorbable sutures were used. A total of 37 patients underwent reoperation for recurrence of the postprandial, upper abdominal complaints. Four of them had recurrence of IH after previous closure with absorbable sutures, 14 had previous closure with non-absorbable sutures, and 1 had a recurrence after closure with staples. In 18 patients with symptom recurrence, there was no perioperative sign of $\mathrm{IH}$. Three of them had a marginal ulcer during gastroscopy. Complete postoperative symptom relief was observed in 146 patients $(77.2 \%)$. For patients in which $\mathrm{IH}$ was present during surgery, $82.8 \%$ had relief of pain postoperatively, as compared to $68.5 \%$ for those procedures in which no IH was found.

\section{Predictors of Internal Herniation During Reoperation and Relief of Symptoms}

There was no significant difference in the presence of IH between females and males ( $p=0.107)$ (Table 3). When internal herniation was visible on $\mathrm{CT}$, IH was found present perioperative in $82.8 \%$ of procedures (OR 4.78; 95\% CI 2.09-10.93). In patients with normal abdominal CT, internal herniation was found in $47.3 \%$ of procedures (OR 0.20 ; 95\% CI 0.09-0.45). In acute surgery, perioperative $\mathrm{IH}$ was seen more frequently than in elective surgery (OR 3.74, 95\% CI 1.91-7.31).

A predictive factor for pain relief after delayed closure of mesenteric defects was IH on CT (OR 4.24, 95\%CI 1.6311.05). Presence of IH perioperatively affected postoperative pain relief (OR 2.21, 95\%CI 1.11-4.40) (Table 4). In multivariate analysis, a positive CT scan for IH was the only independent predictor for pain relief. Time from initial LRYGB to reoperation, the location of $\mathrm{IH}$, and the closure technique did not seem to affect postoperative pain relief. There was no significant correlation between smoking status and postoperative pain relief (Table 4).

\section{Discussion}

A swirl sign on CT was predictive for both perioperative presence of $\mathrm{IH}$ as well as for postoperative pain relief after delayed closure of mesenteric defects. Actual visible IH during laparoscopy appeared more common in an acute setting than when surgery was performed electively. Perioperative presence of $\mathrm{IH}$ was a predictive factor for pain relief postoperative; however, the location of the $\mathrm{IH}$ did not seem to affect postoperative pain relief.

The number of relaparoscopies and performed CT scans for suspected IH increased considerably over the years in our clinics. A possible explanation for this trend may be increased knowledge and awareness regarding long-term complications of LRYGB. The median interval between LRYGB and reoperation for suspected $\mathrm{IH}$ in our study was comparable to other studies [5, 12, 13]. Previous studies reported rapid excess weight loss (EWL) as a predictive factor for the incidence of $\mathrm{IH}$, in which the risk of developing $\mathrm{IH}$ was twice as high in patients with rapid EWL [19, 20]. We have used LOWESS local regression to determine the point on which \% TWL could 
be a predictor for pain relief, which was $40 \%$. In our study, $\%$ TWL $\geq 40 \%$ as compared to a $\%$ TWL $<40 \%$ did not seem to affect the intraoperative presence of IH or of postoperative pain relief. As our study was retrospective, we did not have the exact weight loss per time period whether there was rapid weight loss could not be determined.

The presence of a swirl sign on CT was a predictor for both intraoperative presence of $\mathrm{IH}$ and postoperative symptom relief. However, a varying sensitivity of CT scans for diagnosing IH has been described in literature, ranging between 61 and $83 \%$ [14, 16]. The existence of intermitting IH could be an explanation for these low sensitivity rates, as the CT scans were not always performed at the moment when a patient experiences pain.

Notably, in some patients with a proven IH on CT, there was a long interval between $\mathrm{CT}$ and reoperation. In three patients, CT was performed in a non-bariatric hospital, where they did not acknowledge the pain as IH. Once the patients arrived in one of our institutions, the IH was seen on CT and reoperation was performed soon afterwards. Also, in two patients, symptoms had dissolved at the time of interpretation of the $\mathrm{CT}$ and therefore underwent elective surgery. However, we would advise to perform a reoperation when IH is seen on CT as soon as possible to reduce symptoms and prevent potential incarceration.

We recommend that in all patients with chronic and/or intermittent postprandial, upper abdominal pain, a treatment with PPI and mucosal protective drugs is started. If this does not give pain relief, the presence of cholecystolithiasis should first be excluded by ultrasound. If there are no gallstones detected or if the patient does not have a gall bladder anymore, we would advise to perform a CT scan in order to rule out IH. If there is no swirl sign on CT, gastroscopy should be

Fig. 3 Treatment algorithm for patients with chronic and/or intermittent postprandial upper abdominal pain. (1) Treatment with PPI (proton-pump inhibitor) and MPD (mucosal protective drugs) for gastric irritation, gastritis, or marginal ulcer. (2) If a marginal ulcer is not found during gastroscopy and a CT scan has not been performed yet, a CT scan prior to diagnostic laparoscopy is recommended to exclude other abdominal pathologies

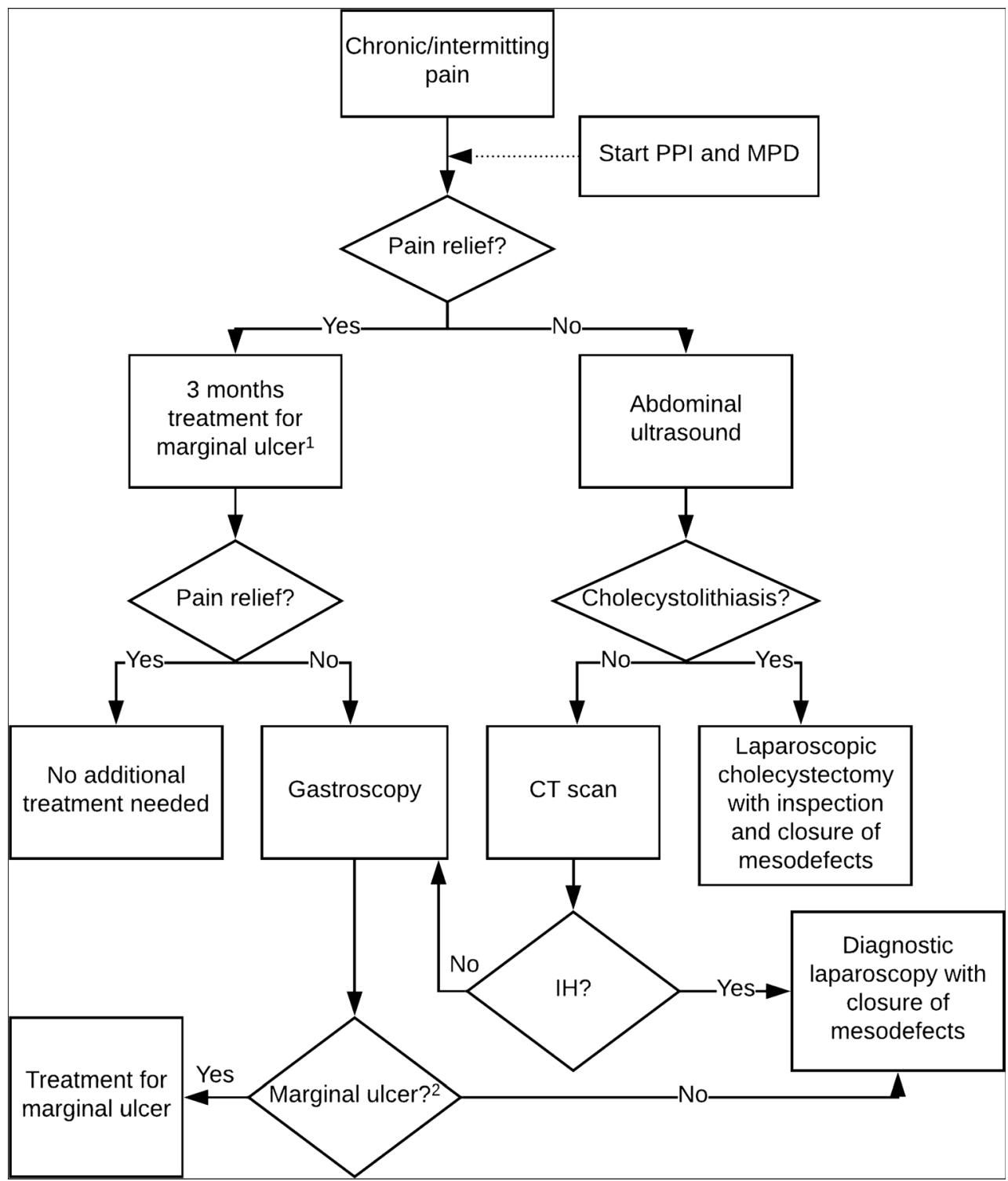


performed to exclude the presence of a marginal ulcer. If the gastroscopy is negative as well and symptoms persist, we would advise to perform a diagnostic laparoscopy to close the mesenteric defects. Our recommended treatment algorithm for chronic and/or intermittent complaints can be found in Fig. 3.

Overall, IH was present during surgery in only $61.3 \%$ of procedures. Surprisingly, $77.2 \%$ of all patients did report postoperative pain relief after closure of mesenteric defects. In $68.5 \%$ of all procedures in which no IH was found perioperatively, postoperative pain relief was reported. Possible explanations for this observation are the intermittent presence of IH or a placebo effect of reoperation.

In our institutions, mesenteric defects were not routinely closed at primary LRYGB. Complications caused by closure of the mesenteric defects such as kinking and adhesions have been reported; however, the incidence of these complications seemed low [6,22]. Especially after Stenberg and colleagues demonstrated the benefits of closure of the defects in their randomised controlled trial, we have decided to routinely close the mesenteric defects at primary LRYGB to reduce the incidence of IH as of January 2017 [21]. In our study, the incidence of IH is relatively low (2.8\%). However, as there is no Dutch database of LRYGB of our study period, it is possible that some patients underwent reoperation for (suspected) IH in other institutions. On the contrary, we have performed reoperation in four patients who did not undergo their LRYGB in one of our institutions. Therefore, our incidence rate is an estimation. Other studies have also shown a lower incidence of IH after closure of the mesenteric defects both with non-absorbable sutures and with staples [23, 24]. However, it has also been reported that even if the mesenteric defects are closed during LRYGB, mesenteric defects might reoccur if patients have excessive weight loss [25]. In the present study, there were 37 patients with a reoperation after closure of the defects. There may have been limited experience with the closing technique of the mesenteric defects when the first relaparoscopies for suspected IH were performed. A learning curve for LRYGB of 100 procedures has been described [26, 27]. To our knowledge, there are no studies describing the learning curve for the closure of mesenteric defects. We noticed that when operating these patients, it might be easier to unravel the herniation by starting counting back from the terminal ileum. In the present study, we could not demonstrate a significant association between year of reoperation and postoperative pain relief; therefore, we expect that the learning curve for the closure of the mesenteric defects may be short.

Closure of mesenteric defects with sutures or with staples during initial LRYGB appears to result in lower incidence of $\mathrm{IH}$ as compared to no closure $[6,20,21]$. In the present study, there is no significant difference in the odds of symptom relief after closure of mesenteric defects with sutures as compared to staples. A limitation of this study is the small number of patients in whom staples were used to close the mesenteric defects. Further research to the difference in the use of staples versus non-absorbable sutures is recommended.

In conclusion, pain relief after closure of the mesenteric defects for (suspected) IH remains unpredictable. A swirl sign on CT was the only significant predictor of pain relief after reoperation for (suspected) IH after delayed closure of mesenteric defects of LRYGB. However, many patients benefit from closure of the mesenteric defects, irrespective of perioperative presence of $\mathrm{IH}$, and therefore, reoperation for suspected IH is recommended if no marginal ulcer was found during gastroscopy.

Acknowledgements We would like to acknowledge T. Martijn Kuijper for his statistical assistance.

\section{Compliance with Ethical Standards}

Conflict of Interest The authors declare that they have no conflict of interest.

Ethical Approval and Informed Consent For this type of study, formal consent is not required.

Open Access This article is distributed under the terms of the Creative Commons Attribution 4.0 International License (http:// creativecommons.org/licenses/by/4.0/), which permits unrestricted use, distribution, and reproduction in any medium, provided you give appropriate credit to the original author(s) and the source, provide a link to the Creative Commons license, and indicate if changes were made.

\section{References}

1. Spivak H, Abdelmelek MF, Beltran OR, et al. Long-term outcomes of laparoscopic adjustable gastric banding and laparoscopic Rouxen-Y gastric bypass in the United States. Surg Endosc. 2012;26(7): 1909-19.

2. Colquitt JL, Pickett K, Loveman E, et al. Surgery for weight loss in adults. Cochrane Database Syst Rev. 2014;8:CD003641.

3. Courcoulas AP, Goodpaster BH, Eagleton JK, et al. Surgical vs medical treatments for type 2 diabetes mellitus: a randomized clinical trial. JAMA Surg. 2014;149(7):707-15.

4. Sjostrom L, Narbro K, Sjostrom CD, et al. Effects of bariatric surgery on mortality in Swedish obese subjects. N Engl J Med. 2007;357(8):741-52.

5. Iannelli A, Buratti MS, Novellas $\mathrm{S}$, et al. Internal hernia as a complication of laparoscopic Roux-en-Y gastric bypass. Obes Surg. 2007;17(10):1283-6.

6. Aghajani E, Jacobsen HJ, Nergaard BJ, et al. Internal hernia after gastric bypass: a new and simplified technique for laparoscopic primary closure of the mesenteric defects. J Gastrointest Surg. 2012;16(3):641-5.

7. Quezada N, Leon F, Jones A, et al. High frequency of internal hernias after Roux-en-Y gastric bypass. Obes Surg. 2015;25(4): 615-21.

8. Higa KD, Ho T, Boone KB. Internal hernias after laparoscopic Roux-en-Y gastric bypass: incidence, treatment and prevention. Obes Surg. 2003;13(3):350-4. 
9. Himpens J, Verbrugghe A, Cadiere GB, et al. Long-term results of laparoscopic Roux-en-Y gastric bypass: evaluation after 9 years. Obes Surg. 2012;22(10):1586-93.

10. Garza Jr E, Kuhn J, Arnold D, et al. Internal hernias after laparoscopic Roux-en-Y gastric bypass. Am J Surg. 2004;188(6):796800 .

11. Agaba EA, Gentles CV, Shamseddeen $\mathrm{H}$, et al. Retrospective analysis of abdominal pain in postoperative laparoscopic Roux-en-Y gastric bypass patients: is a simple algorithm the answer? Surg Obes Relat Dis. 2008;4(5):587-93.

12. Danshoj Kristensen S, Jess P, Karen Floyd A, et al. Internal herniation after laparoscopic antecolic Roux-en-Y gastric bypass: a nationwide Danish study based on the Danish National Patient Register. Surg Obes Relat Dis. 2016;12(2):297-303.

13. Al-Mansour MR, Mundy R, Canoy JM, et al. Internal hernia after laparoscopic antecolic Roux-en-Y gastric bypass. Obes Surg. 2015;25(11):2106-11.

14. Lockhart ME, Tessler FN, Canon CL, et al. Internal hernia after gastric bypass: sensitivity and specificity of seven $\mathrm{CT}$ signs with surgical correlation and controls. AJR Am J Roentgenol. 2007;188(3):745-50.

15. Chowbey P, Baijal M, Kantharia NS, et al. Mesenteric defect closure decreases the incidence of internal hernias following laparoscopic Roux-en-Y gastric bypass: a retrospective cohort study. Obes Surg. 2016;26:2029-34.

16. Goudsmedt F, Deylgat B, Coenegrachts K, et al. Internal hernia after laparoscopic Roux-en-Y gastric bypass: a correlation between radiological and operative findings. Obes Surg. 2015;25(4):622-7.

17. Cho M, Pinto D, Carrodeguas L, et al. Frequency and management of internal hernias after laparoscopic antecolic antegastric Roux-en$\mathrm{Y}$ gastric bypass without division of the small bowel mesentery or closure of mesenteric defects: review of 1400 consecutive cases. Surg Obes Relat Dis. 2006;2(2):87-91.
18. O'Rourke RW. Management strategies for internal hernia after gastric bypass. J Gastrointest Surg. 2011;15(6):1049-54.

19. Schneider C, Cobb W, Scott J, et al. Rapid excess weight loss following laparoscopic gastric bypass leads to increased risk of internal hernia. Surg Endosc. 2011;25(5):1594-8.

20. Geubbels N, Roell EA, Acherman YI, et al. Internal herniation after laparoscopic Roux-en-Y gastric bypass surgery: pitfalls in diagnosing and the introduction of the AMSTERDAM classification. Obes Surg. 2016;26:1859-66.

21. Stenberg E, Szabo E, Agren G, et al. Closure of mesenteric defects in laparoscopic gastric bypass: a multicentre, randomised, parallel, open-label trial. Lancet. 2016;387(10026):1397-404.

22. Kristensen SD, Floyd AK, Naver L, et al. Does the closure of mesenteric defects during laparoscopic gastric bypass surgery cause complications? Surg Obes Relat Dis. 2015;11(2):459-64.

23. Delko T, Kraljevic M, Kostler T, et al. Primary non-closure of mesenteric defects in laparoscopic Roux-en-Y gastric bypass: reoperations and intraoperative findings in 146 patients. Surg Endosc. 2016;30(6):2367-73.

24. Aghajani E, Nergaard BJ, Leifson BG, et al. The mesenteric defects in laparoscopic Roux-en-Y gastric bypass: 5 years follow-up of non-closure versus closure using the stapler technique. Surg Endosc. 2017;31(9):3743-8.

25. Hope WW, Sing RF, Chen AY, et al. Failure of mesenteric defect closure after roux-en-Y gastric bypass. JSLS. 2010;14(2):213-6.

26. Schauer P, Ikramuddin S, Hamad G, et al. The learning curve for laparoscopic Roux-en-Y gastric bypass is 100 cases. Surg Endosc. 2003;17(2):212-5.

27. Pournaras DJ, Jafferbhoy S, Titcomb DR, et al. Three hundred laparoscopic Roux-en-Y gastric bypasses: managing the learning curve in higher risk patients. Obes Surg. 2010;20(3):290-4. 